\title{
FUNDAMENTALS OF THE SYNCHROTRON RADIATION FACILITY CONSTRUCTION
}

\author{
M. Eriksson \\ MAX-lab, Box 118, 22100 Lund, Sweden
}

\begin{abstract}
Some fundamental properties of synchrotron radiation and electron beam characteristics in synclirotron radiation storage rings are recapitulated. Then, different design criteria for the construction of synchrotron radiation facilities are discussed as well as some technical solutions to the problem of matching the light and electron beam properties.
\end{abstract}

PACS numbers: $41.60 . \mathrm{Ap}$

\section{Introduction}

We have now more than fifty synchrotron radiation facilities in the world. Few of them resemble each other but their parameters values vary quite a lot. Each of them was designed to meet the actual demands and resources. The trend has gone towards specialization rather than to construct general purpose machines.

The development of new synchrotron light sources, that is different kinds of undulators and wigglers, has drastically changed the design criteria of the storage rings. This process is still strongly in progress and we are now facing new developments in this area.

When designing a synchrotron radiation storage ring the most important and difficult part is to put down a specification list. This will reflect the user community needs, the ambition level and the amount of money available. Once this is done, the iteration process between the machine design proposals and the specifications starts.

Therefore, the first questions to be put are the following:

a. Which is (are) the most important spectral region(s)?

b. What are the light characteristic demands and priorities concerning flux, brilliance and time structure?

c. Ilow many and what kind of beamlines do you want?

d. What boundary conditions are we facing concerning money, personnel, space etc.?

After the first iteration, you will probably find an inconsistency between the answers to the points above. Now, the real interesting discussion starts: 
a. Can we sacrifice some non-top priorities? If you do not have the ambition of building the best facility in the world (which it probably will be for a very limited time period), you probably can and cooperate with other labs for the things you will not get.

b. Can we find some new ideas to solve technical problems in a more efficient and cheaper way?

If this homework is done properly, you will probably save a lot of problems and bad surprises when starting the construction.

\section{Figures of merit}

There are mainly two properties characterising an SR source. The first is the flux defined as

$$
\Phi=\frac{N_{\mathrm{f}}}{0.1 \%[\mathrm{mrad}]} \quad \text { [photons } /(\mathrm{s}, 0.1 \% \text { energy spread, mrad horizontally)]. }
$$

The brilliance $B$ is the peak flux density in phase space

$$
B=\frac{N_{\mathrm{f}}}{0.1 \%\left[\mathrm{~mm}^{2}\right]\left[\mathrm{mrad}^{2}\right]} .
$$

The flux is a function of electron current and electron encrgy only. When calculating the brilliance we have to take into account the phase space defined by the diffraction properties and that given by the electron beam emittance.

Other figures of merit for an SR ring are electron beam lifetime, spatial stability, ring reliability and ease of operation.

\section{Specification goals}

\subsection{Spectral region}

For a dipole source, the critical wavelength where intensity is close to maximum, is given by

$$
\lambda_{\mathrm{c}}=\frac{18.6}{E^{2} B}[\AA]
$$

where $E$ is the electron energy in GeV and $B$ the magnet field in T (Fig. 1).

We should thus choose the electron energy to cover the photon spectral region of interest, but a too high electron cnergy might induce unnecessary heat problems and a too high ring cost. We must also take into account that a large bending magnet radius will put the optical elements further away which will reduce the opening angle for a given optical clement size.

The use of insertion devices and then especially undulators puts even tighter boundary conditions on the electron energy. The spectral region is given by the undulator period and its magnet field. The total flux is proportional to the number of undulator periods. The latter is strongly dependent on the minimum undulator gap allowed in the machine.

As a consequence of this, the optimization process of for instance a third generation storage ring for SR is heavily dependent on the cvolution of the insertion 


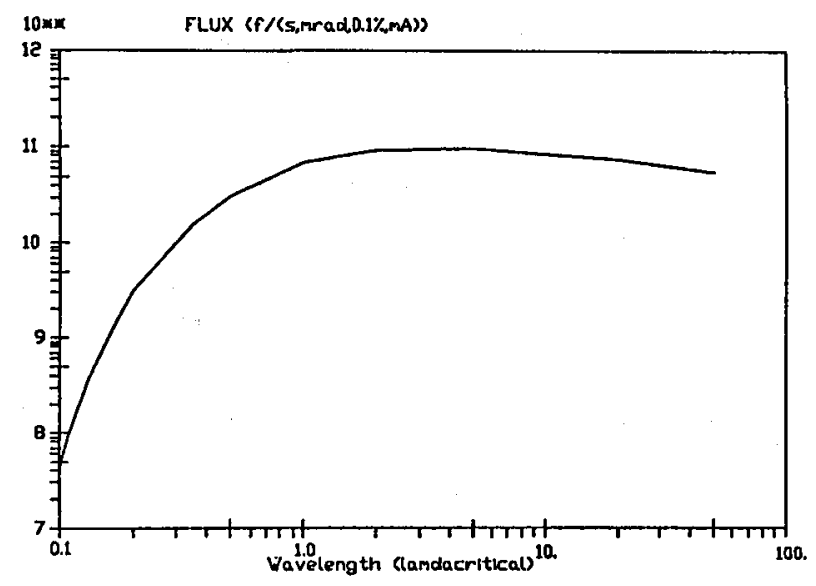

Fig. 1. Synchrotron radiation spectral distribution.

devices. A ring optimised for an undulator minimum gap of $40 \mathrm{~mm}$, which was a conservative value some years ago, is certainly quite different from a ring optimised for a $10 \mathrm{~mm}$ gap, which is used and discussed today.

\subsection{Malching}

The photon angular spread defined by diffraction can generally be approximated for Gaussian distributions to

$$
\sigma_{\mathrm{ph}}^{\prime}=\sqrt{\frac{\lambda}{2 L}}
$$

where $\sigma^{\prime}$ is the root-mean-square angular spread, $\lambda$ - the emitted light wavelength and $L-$ the source length.

The diffraction relation for Gaussian distributions

$$
\sigma_{\mathrm{ph}} \sigma_{\mathrm{ph}}^{\prime}=\frac{\lambda}{4 \pi}
$$

yields the apparent source size $\sigma_{\mathrm{ph}}=\frac{1}{\pi} \sqrt{\lambda L / 8}$.

The electron beam phase space area or emittance $\varepsilon=\sigma_{\mathrm{e}} \sigma_{\mathrm{e}}^{\prime}$ should thus preferably be smaller than $\lambda / 4 \pi$. If this is the case, the source is called diffraction-limited. This demand is so far difficult to fulfil, especially for shorter wavelengths.

Even if the electron beam emiltance is smaller than $\lambda / 4 \pi$, we need to match the phase space form of the electron beam emittance to that induced by diffraction. The mismatch between these phase-space forms for a dipole source is seen in Fig. 2, where $L$ is in the $\mathrm{mm}$ range which gives $\sigma_{\mathrm{ph}}$ in the $\mu \mathrm{m}$ range while the electron beam size is orders of magnitudes larger. For an undulator, with $L$ in the meter region, $\sigma_{\mathrm{ph}}$ is some orders of magnitude larger which yields a better match to the electron beam size. This fact plus the larger number of emitting sources explain the high brilliance attainable from an undulator in a low emittance storage ring. 


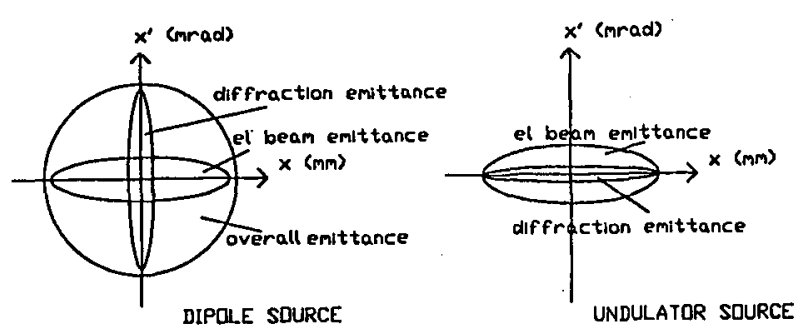

Fig. 2. Photon and electron beam emiltances in phase space.

\section{Magnet lattice}

\subsection{Belalron molion}

A ring magnet lattice consists mainly of dipole magnets which recirculate the electron beam and magnet lenses, quadrupole magnets. These lenses will force the stored particles to oscillate around a reference orbit. If we smear out the focusing evenly around the ring, we should obviously get a harmonic oscillation for a certain particle

$$
x(\Psi)=C \sin (K \Psi+\Phi),
$$

where $C$ is the oscillation amplitude, $K$ is the wave number, $\Psi-$ the azimuth angle in the ring and $\Phi$ - a phase angle.

In a real machine, we have piece-wise constant focusing lenses. A solution to the particle motion can then be described as

$$
x(s)=C \sqrt{\beta(s)} \sin (Q \Psi(s)+\Phi) .
$$

$\beta(s)$ is now a modulating function, $s-$ the coordinate in the reference orbit direction and $Q$ is the betatron wave number defining the number of oscillations the particle will execute during one turn in the machine. $\Psi$ is now not exactly the azimuth angle but is generally rather close to it. The angle between the particle motion and the reference orbit is achicved by differentiating $x$ with respect to $s$ :

$$
x^{\prime}(s)=\frac{\mathrm{d} x(s)}{\mathrm{d} s} .
$$

In phase space $x, x^{\prime}$ the particle will now describe an ellipse when moving in the ring. The oscillation amplitudes for the particles in the beam will have a Gaussian distribution. The particle with the root-mean-square (RMS) amplitude will have its motion described by

$$
x(s)=\sqrt{\varepsilon \beta(s)} \sin (Q \Psi(s)+\Phi),
$$

where $\varepsilon$ defining the oscillation amplitude is the area (divided by $\pi$ ) of the phase space ellipse and is called the electron beam emittance.

We have two directions of oscillations, the radial $(x)$ and vertical $(y)$ ones, therefore we now have two $\beta$ functions.

One example of the $\beta$ functions is seen for the MAX I ring for one cell in Fig. 3. We can thus read the beam size variations around the machine. One 


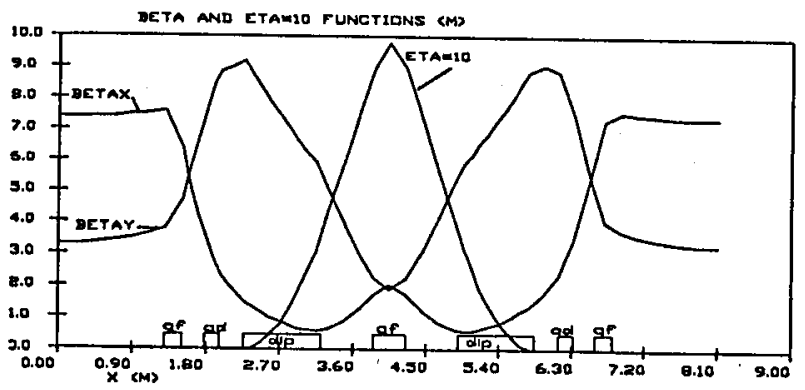

Fig. 3. Beta and dispersion (eta) functions for a double bend achromat.

peculiarity is that the $\beta$ function is at maximum at focusing lenses and minimum at defocusing ones. Since the sign of focusing is changing when going from radial to vertical motion, the beam size has a horizontal maximum where the vertical size is at minimum and vice versa.

\subsection{Dispersion}

A particle with a higher energy than the nominal one will have a larger bending radius in a dipole magnet field. An achromatic bend is seen in Fig. 4. A particle with a higher energy is entering the first dipole magnet from the left on the reference axis. This particle is then deflected less than the nominal one in this

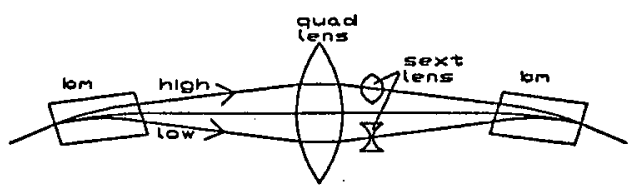

Fig. 4. Double bend achromat showing particles of ligh, nominal and low energy.

magnet but will then enter a focusing quadrupole which will deflect the particle inwards the machine. By symmetrical reasons, the particle will, after the second dipole transit, be situated on the reference orbit again. The function, describing the position of an off-energy particle with a momentum deviation $\Delta P / P=1$, is called the dispersion function $\eta$ and this is also seen in Fig. 3.

\section{Radiation damping and beam emittance}

As we have seen above, the particles stored in the ring execute oscillations around a reference orbit. Let us first look for the radiation damping in the vertical direction as seen in Fig. 5.

An oscillating particle will emit a photon in a dipole magnet. The recoil of the photon will have one longitudinal component retarding the particle and one vertical component damping the vertical oscillation. The longitudinal recoil will be 


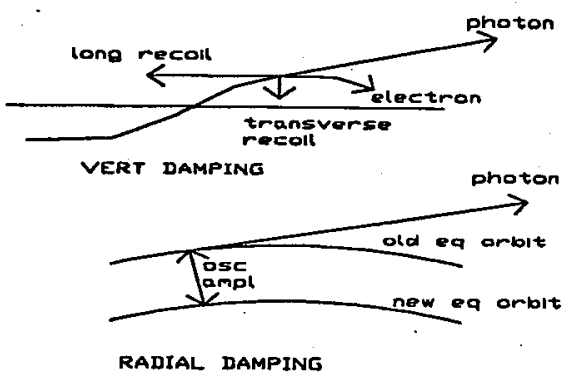

Fig. 5. Vertical and radial damping.

replaced by the accelerating cavity while the vertical recoil component will damp the oscillation.

The situation is somewhat more complicated in the radial direction. Let us look at Fig. 5 where we assume that a particle of nominal energy on the reference orbit emits a photon in a bending magnet. Apart from the damping mechanism described for the vertical case above, the particle will now suddenly have another equilibrium orbit introduced by its changed energy and the present non-zero dispersion. The introduced oscillation amplitude equals the particle distance to the new equilibrium orbit.

This mechanism is in fact responsible for the equilibrium emittance in a storage ring. We can also see that a large dispersion in the bending magnets introduces large oscillation amplitudes and thus a larger emittance.

\section{Decreasing the electron bcam emittance}

The chase is now for smaller dispersion functions in the bending magnets in order to decrease the particle beam emittance and thus increase the brilliance. The first thing is of course to enlarge the number of achromats to get a small dispersion in the bending magnets. The smaller the bending angle of the bending magnet, the smaller the dispersion. We can also lower the electron beam energy and get a smaller recoil. The emittance is in fact scaling like

$$
\varepsilon \propto E^{2} / N^{3},
$$

where $E$ is the electron energy and $N-$ the number of bending magnets.

We cannot, however, push this scheme too far. The reason for this is chromaticity corrections. A particle of higher energy will expcrience a weaker focusing in the quadrupole lenses and should thus get a lower betatron wave number $Q$. The chromaticity

$$
\xi=\frac{\mathrm{d} Q}{\mathrm{~d} P / P}
$$

will then be negative which will excite the head-tail instability. We correct the chromaticity by introducing nonlinear lenses, sextupole magnets, at places with non-zero dispersion as seen in Fig. 4. The particle position is here energy-dependent and the nonlinear sextupole lens can then compensate the betatron $Q$-value. 
For small dispersion functions, we need stronger sextupoles and this will make the lattice more unstable, the dynamic aperture is reduced. This mechanism is one factor limiting the possibility to reach small remittances.

\section{One lattice example}

The machine functions of the MAX II ring is seen in Fig. 6. First, we notice that the cell is not strictly an achromat. The dispersion function is not zero at the long straight sections. The reason for this is that the mean dispersion in the bending magnets (where the emittance is defined) can be minimised by allowing dispersion at both dipole ends. We can in principle adjust the dispersion to zero

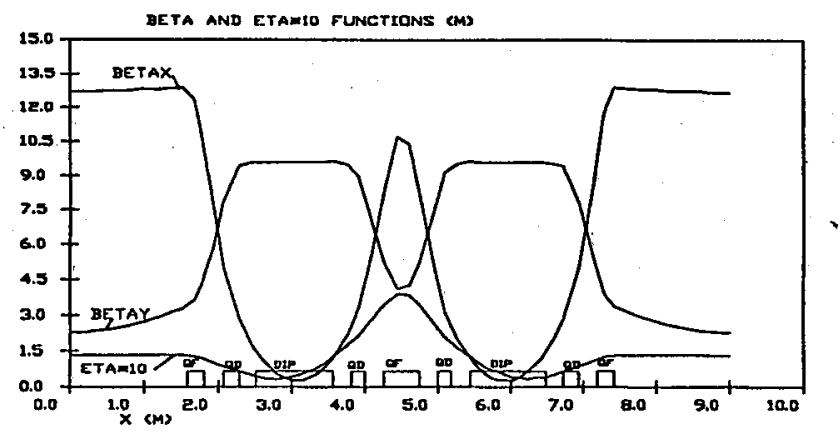

Fig. 6. MAX II beta and eta functions.

in the middle of the bending magnet which should effectively double the number of dipoles compared to the conventional double achromat example and thereby reducing the emittance by a factor of eight.

Another feature is that no sextupoles are seen in the lattice. The sextupoles are integrated into the quadrupole lenses by a special machining of the magnet iron surfaces. This yiclds an optimum sextupole distribution with a large momentum acceptance and a large dynamic aperture paired with a compact and cheap magnet lattice. Since we simultancously restrict the tuning flexibility, we have to carefully calculate the lattice characteristics prior production.

\section{Electron beam lifetime}

For small emittance storage rings, we have mainly two effects defining the electron beam lifetime.

We have two loss mechanisms given by vacuum.

1.a. The first one is the bremsstrahlung effect. Electrons are inelasticly scattered by rest gas molecules with bremsstrahlung emission as a consequence. If the energy lost by the electron is sufficiently large, this electron will be lost. This effect will be more severe the higher the particle energy is, since the relative energy loss increases with energy. 
1.b. The other vacuum loss mechanism is the elastic scattering process. An electron is then deflected by a rest gas molecule and starts to oscillate around the reference orbit. If the oscillation amplitude is small enough, that is the impact parameter at the scattering event is large enough, the electron will avoid hitting the smallest ring aperture and the oscillation will be damped by the synchrotron radiation damping. This damping time is typically some $10 \mathrm{~ms}$ or some 10000 turns in the ring. If the scattering angle is too large, the electron will find the smallest aperture in the ring during this large number of turns and be lost. The elastic scattering effect will in fact define the minimum undulator gap accoptable. By a careful vacuum system design we can thus influence the undulator gap choice. The elastic scattering loss process decreases quadratically with clectron energy and gap size.

2. The next loss mechanism is the Touschek effect. In this case, electrons are scattered against each other within the electron bunch. Transverse oscillation energy can then be transferred to the longitudinal direction. If this energy transfer is large enough, the electrons might be scattered out from the ring energy acceptance. As we try to decrease the electron beam emittance, we increase the electron collision rate. Since the Touschek effect is strongly energy dependent, this loss mechanism is most pronounced at low energy rings.

\section{Magnets}

We have briefly discussed some lattice aspects above. We have mainly two types of magnets in the lattice, dipoles and quadrupoles.
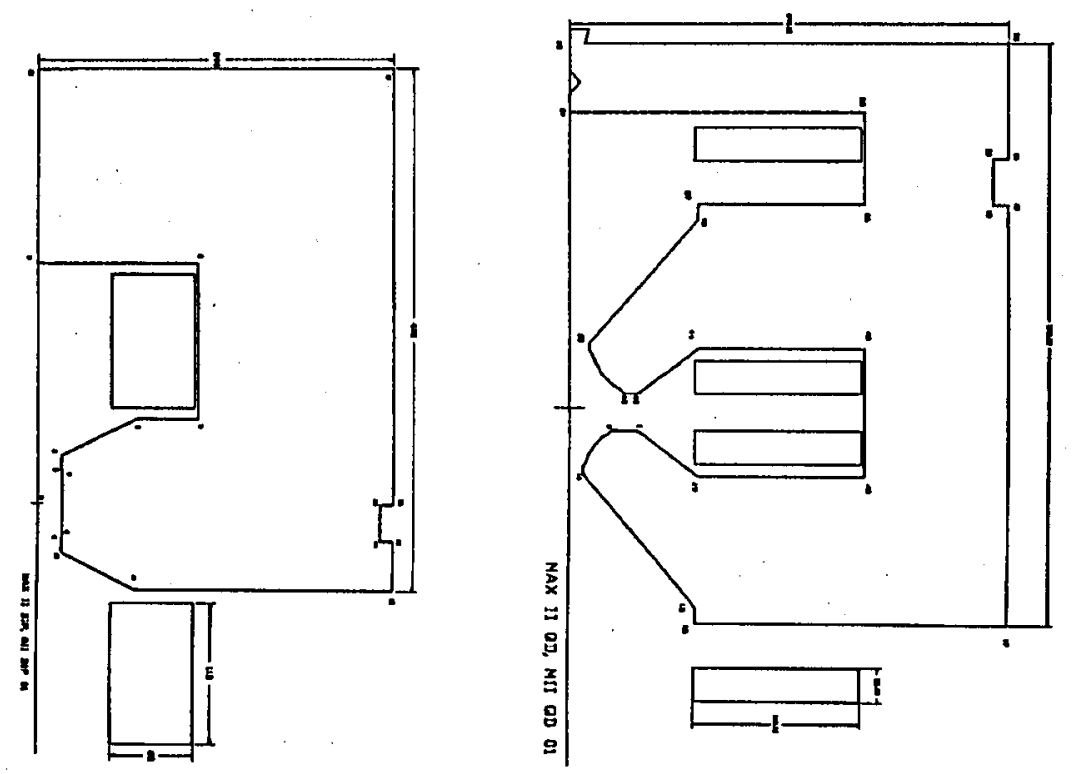

Fig. 7. MAX II dipole and quadrupole magnets. 
As an example, we show these linds of magnets for the MAX II ring (Fig. 7). These magnets consist of laminated steel, the laminates are glued together. The laminates are stamped from big steel sheets. The grooves at the top of the magnets are used when aligning the magnets. These grooves fit exactly to machined knobs on the girder therefore a complete cell can then be manufactured outside the lab including vacuum system and otlicr details.

The quadrupole magnets contain also the sextupole components necessary for chromatic correction. The quadrupole and sextupole ficld strengths are thus coupled. Eventual fine tuning of the chromaticity is done with smaller correction magnets.

\section{Vacuum}

As mentioned above, we need a good vacuum to get a sufficiently long electron beam lifetime. The scattering effects are strongly dependent on the rest gas $Z$ number, therefore we do not care so much about hydrogen but must avoid heavier elements.

The background pressure is seldom a problem in electron storage rings. More severe is the synchrotron radiation induced desorption. This is a two-step process. Photoelectrons are emitted by the synchirotron radiation. These electrons desorb twice, once when the electrons leave the metal surface and another time when they hit the surface again. The gas released is mostly $\mathrm{CO}, \mathrm{CO}_{2}$ and $\mathrm{CII}_{4}$. After a sufficiently long beam conditioning period, that is hundreds of Ah (ampere-hours), the system is generally well cleaned.

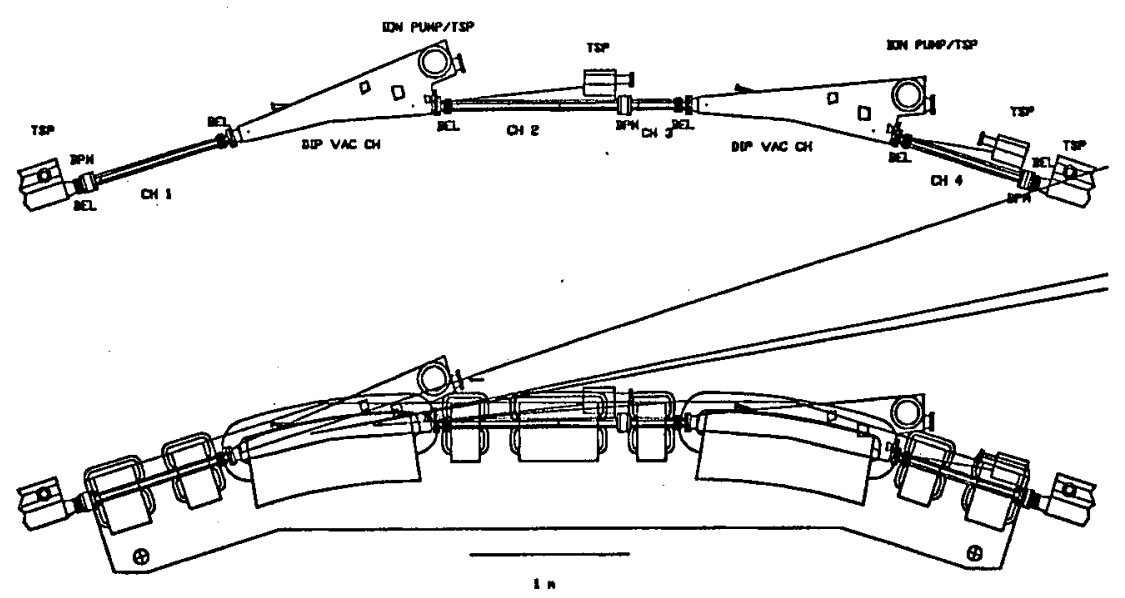

Fig. 8. MAX II vacuum system.

The narrow band within which the synchrotron light is striking the vacuum chamber is conditioned pretty quickly. The surfaces where the photoelectrons hit the chambers are much larger and these surfaces will define the conditioning period 
length. By choosing a proper radiation absorber geometry, the gassing rate and the commissioning time can be strongly influenced.

The vacuum system must be thoroughly cleaned prior mounting. As pumps, we generally use ion pumps which are clcan and efficient. Lately NEG (non-evaporable getter) pumps have been used and cheap alternative is offered by the TSP (titan-sublimation pump) pumps which effectively pump the gases desorbed.

We see below the vacuum system for a MAX II cell (Fig. 8). The radiation hits the absorbers placed close to the vacuum pumps. The vacuum system is uncoupled to the magnets therefore the magnets can be repaired without breaking the ring vacuum.

\section{Radio fiequency (RF) acceleration}

The power lost in synclirotron radiation must be compensated for the electrons. The power needed increases with the fourth power of the electron energy for a given bending radius and the power emitted might range to several $100 \mathrm{~kW}$ in high energy rings.

Apart from this, we must also create a potential well of sufficient depth to keep the Touschek-scattcred particles in the machine. To get a sufficiently high accelerating voltage, we use a tuned circuit resembling an ordinary tuned (LC) circuit, an RF cavity. RF power is fed into the cavity where high alternating electric fields are created. The voltage induced is shown in Fig. 9. The frequency used

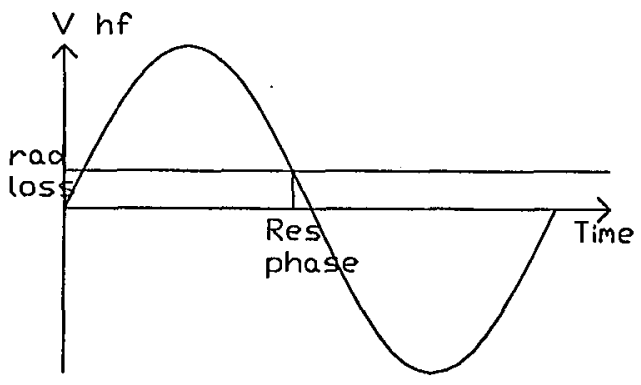

Fig. 9. RF accelcration.

is typically 350-500 MHz, but as low frequencies as $50 \mathrm{MHz}$ are used, especially at low energy rings. The cavity voltage induced is much higher than the synchrotron radiation energy lost each turn in the ring. An electron of nominal energy entering the cavity at such a phase that the voltage seen by the electron equals the radiation energy loss will be situated at the equilibrium phase and energy. An electron of too high energy will take a longer path in the ring and arrive later than the nominal one to the cavity. The cavity voltage has then decreased and the electron will then get a smaller energy contribution. If the particle energy is still too high, the electron will arrive even later the next turn and get even less voltage. Sooner or later, the electron will reach the nominal energy but now it is out of phase. It will still lose energy, but when doing so it will walk back to the proper phase. This 
electron oscillation around the equilibrium phase and energy is called synchrotron oscillation and is an automalic process responsible for energy stabilization. The synchrotron radiation will moreover damp this oscillation since particles of higher energy radiate more energy than those of low energy. As a result, the electrons will be bunched with a separating distance equalling the RF wavelength.

The ring circumference must then equal an integer number of $R F$ wavelengths. This number of RF wavelengths defines the maximum number of bunches, or buckets, in the ring. We do not necessarily populate all of these buckets.

\section{Injectors}

We need of course an electron source. This is the electron gun where the electrons are generally emitted from a hot surface. The clectrons are then accelerated in an electric field of some $100 \mathrm{kV}$.

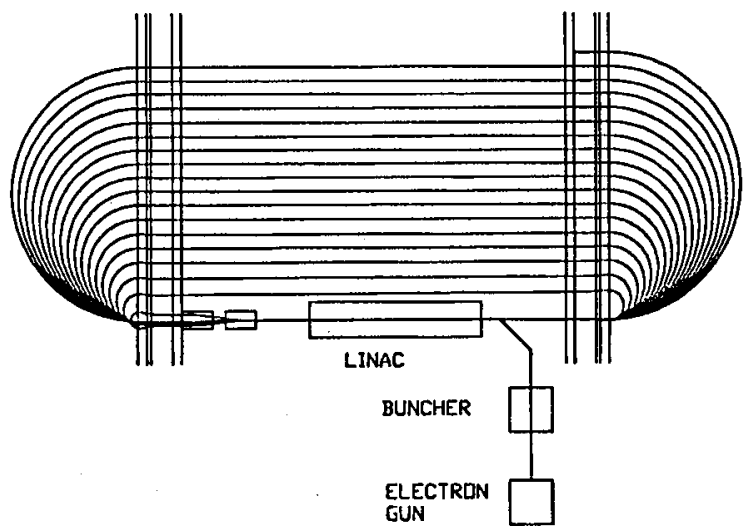

Fig. 10. $100 \mathrm{MeV}$ injector race-track microtron.

The electrons are then accelerated in a pre-accelerator, a race-track microtron or a linear accelerator.

The linear accelerator, or linac, consists of a row of cavities, generally operated at 3 GIIz. The electrons are then injected into a booster synchrotron or directly into the storage ring. The microtron consists of a short linear accelerator and two $180^{\circ}$ bending magnets (Fig. 10). The electrons are then recycled by these magnets and the linac structure is used several times.

If a full-energy booster synchrotron is used or if we inject directly into the storage ring depends on scveral things. Of course, we prefer full energy injection if we can afford it. If not, we can accelerate in the ring after injection.

At injection, the electron beam is shot into the ring (or booster) some distance from the equilibrium orbit. The injected electrons will then oscillate around this orbit and the oscillation amplitude will decrease due to the synchrotron radiation losses. When the injected beam has contracted, we can inject the next beam outside the stored one. In this way, we can stack to rather high currents. 
The booster synchrotron is a simple storage ring where we can ramp the particle energy pretty fast. In the booster, we just inject one pulse per ramping cycle and inject into the storage ring at full energy.

\section{System design considerations}

A total synchrotron radiation facility is a rather complex system including beamlines, experimental stations, building, service etc. Everything must then be squeezed within the boundary conditions given in terms of money, space etc. Technical solutions chosen in one field will generally influence others. We will try to give some examples below.

1. Electron beam position monitors. With modern electronics one can now reach very precise monitoring of the beam positions. This leads to a reduced vacuum aperture need which in its turn reduces cost for magnets, vacuum system and power supplies.

2. By using large numeric machines for the girder production, one can align the magnets very precisely which also turns out to be cost-effective.

3. Aperture defining parameters like vacuum and lattice properties will define the operating electron energy for a given spectral range.

4. The hall floor is another important item. Mistakes will be costly and many people can tell you how not to do, but hardly anyone can tell you the ideal receipt.

5. Reliability. It is very tempting to choose advanced and complicated solutions to meet all the various demands put up. One might have to pay for this in terms of a prolonged commissioning period and reduced reliability.

6 . Flexibility. Cheap solutions are sometimcs sacrificed since they might reduce the machine flexibility. But do we in reality have any flexibility? Can we operate a facility with relaxed parameter values without loosing the motivation for the project?

\section{One facility example}

As an example of what we have discussed so far, we start with the MAX I facility (Fig. 11). As an injector, a $100 \mathrm{MeV}$ race-track microtron is used. Stacking in the $550 \mathrm{MeV}$ storage ring takes thus place at $100 \mathrm{MeV}$, which is a rather low injection energy. Some $300 \mathrm{~mA}$ can be injected and some $200 \mathrm{~mA}$ will generally survive the ramping to $550 \mathrm{MeV}$.

The MAX I ring was operational in 1986. In 1989 it was decided to add a third generation VUV ring, MAX II to the system. The MAX I ring will then be used as injector for MAX II and the two rings will after commissioning in 1995 be operated in parallel (Fig. 12). 


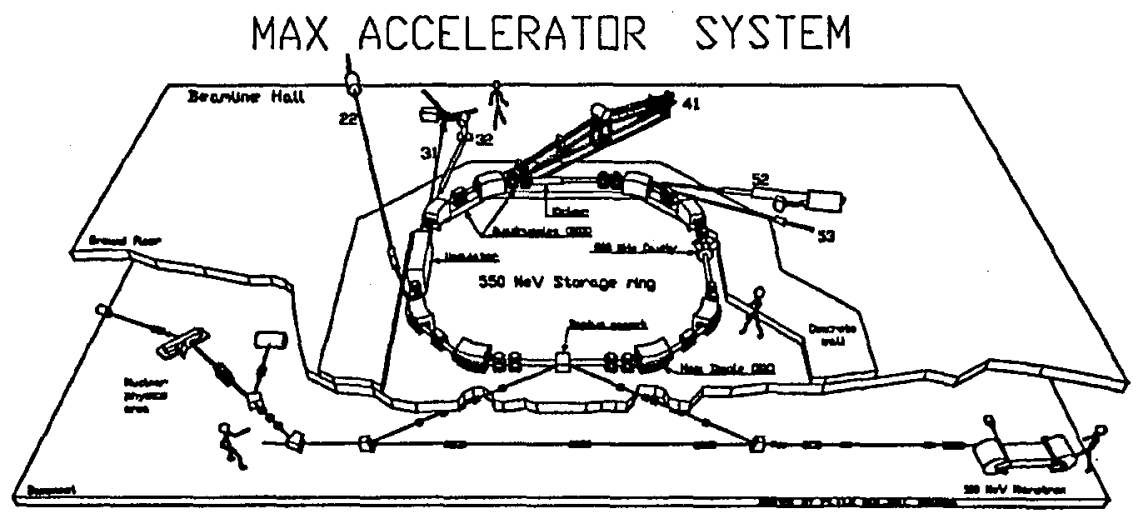

Fig. 11. The MAX I storage ring.

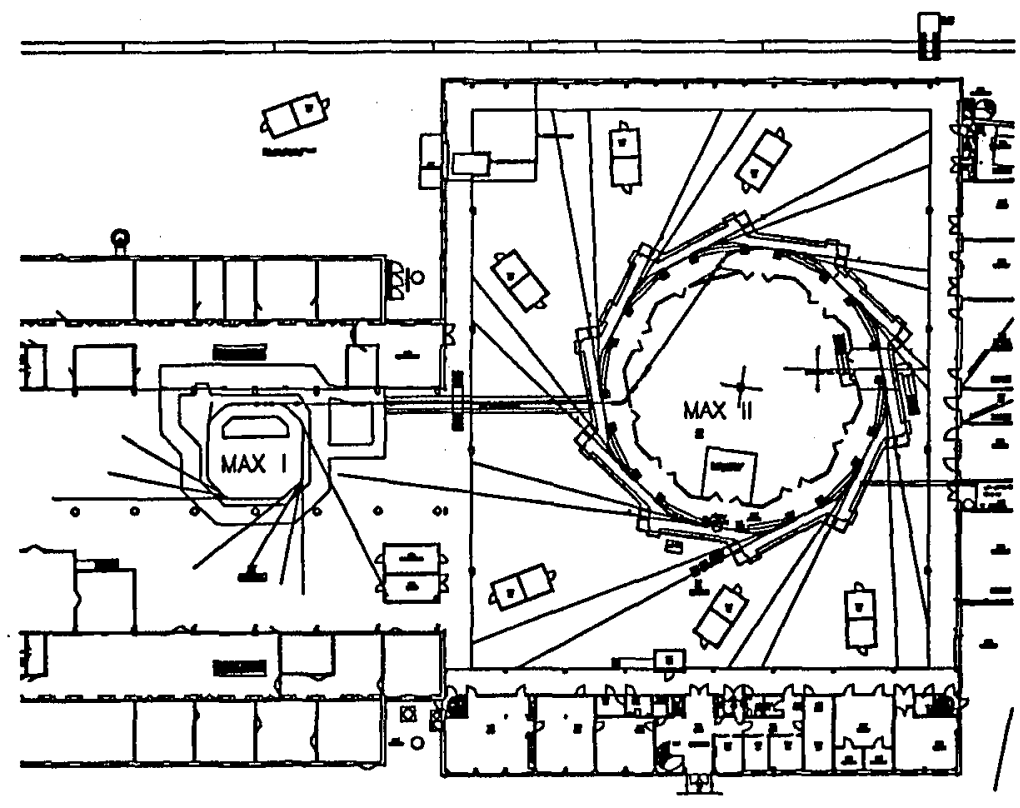

Fig. 12. The two MAX storage rings. 